International Mathematical Forum, Vol. 8, 2013, no. 22, 1091 - 1101

HIKARI Ltd, www.m-hikari.com

http://dx.doi.org/10.12988/imf.2013.3486

\title{
Modeling Traffic Flow for Two and Three Lanes through Cellular Automata
}

\section{B. Luna-Benoso}

Instituto Politécnico Nacional, Escuela Superior de Cómputo, Av. Juan de Dios Batíz, esq. Miguel Othón de Mendizábal, México City 07738, México blunabenoso@yahoo.com

\section{J. C. Martínez-Perales}

Instituto Politécnico Nacional, Escuela Superior de Cómputo, Av. Juan de Dios Batíz, esq. Miguel Othón de Mendizábal, México City 07738, México pepe_cruzm@hotmail.com

\section{R. Flores-Carapia}

Instituto Politécnico Nacional, Centro de Innovación y Desarrollo Tecnológico en Cómputo, Av. Juan de Dios Batíz, esq. Miguel Othón de Mendizábal, México City 07738, México rfcarapia@yahoo.com

Copyright (c) 2013 Benjamín Luna Benoso et al. This is an open access article distributed under the Creative Commons Attribution License, which permits unrestricted use, distribution, and reproduction in any medium, provided the original work is properly cited.

\begin{abstract}
This paper describes two new models for vehicular traffic flow multilane. One applied to vehicular traffic flow for two lanes and one to three lanes. The models are compared with model Rickert, Nagel, Schreckenberg and Latour (RNSL) for the case of two lanes, and the model Daoudia and Moussa (D-M) for the case of three lanes. The models were compared using the parameters of the fundamental diagram and considering the effect of slow vehicles.
\end{abstract}

Keywords: Cellular automata, vehicular traffic flow, fundamental diagram 


\section{Introduction}

At present different models of vehicular traffic flow in terms of the theory of Cellular Automata (CA) have been made [4], [5], [6]. This approach began in 1992 with Nagel and Schreckenberg [2]. They proposed a model for vehicular traffic flow of a single lane probabilistic (NaSch model), which is carried out under four rules. The model NaSch reproduces phenomena encountered in the actual flow of traffic jam as ghosts, however, the model is minimal in the sense that any simplification of some of the rules does not reproduce realistic results. Moreover, the traffic flow is generally composed of various types of vehicles with different desired speeds and moving in more than one lane. If the model NaSch considered various types of vehicles, is the result of creation of platoons, that means slow vehicles are followed by rapid vehicles reducing the average speed of the free flow system slow vehicles. In 1995 Rickert, Nagel, Schreckenberg and Latour published a model of vehicular traffic flow (RNSL model) in two adjacent lanes and independent, and this they added rules allowing the simulation of lane change [3]. From this starts the study of the flow of multi-lane traffic. Multi-lane models are currently studied in greater depth because these are closer to what happens to the flow of traffic in a city. In 2003 Daoudia and Moussa proposed a model for three-lane traffic flow through CA (D-M model) based on the model NaSch on which analyzed the effect of slow cars system [1].

This paper presents the proposal of two models of traffic flow: two and three lanes through cellular automata. There are two types of vehicles each one with different speeds. Analyzes the effect of slow vehicles in a heterogeneous system and compared to the model RNSL for the case of two lanes, and with DM model for the case of three lanes parameters via basic diagram.

\section{NaSch Model}

NaSch model is defined on a probabilistic CA. The model is defined on a 1dimensional array of $L$ cells, cell or sites with periodic boundary conditions. Each cell represents a division of the road of $7.5 \mathrm{~m}$. Each site can be empty or occupied by a car. Each car has an associated speed $v_{i}$ whole between 0 and $v_{\max }=5(\approx 135 \mathrm{~km} / \mathrm{h})$, the maximum speed that can reach cars. There is a variable that determines the progress of all cars known as gap, which represents the total number of empty sites in front of a car. That is, if $x_{i}$ represents the $i$-th position of the vehicle, then $\operatorname{gap}(i)=x_{i+1}-x_{i}-1$. In each discrete time step, the system is composed of $N$ cars updated in parallel according to the following rules:

Step 1: acceleration: 


$$
v_{i}=\min \left(v_{i}+1, v_{\max }\right)
$$

Step 2: braking:

$$
v_{i}=\min \left(v_{i}, \operatorname{gap}(i)\right)
$$

Step 3: randomness:

$$
v_{i}=\max \left(v_{i}-1,0\right) \text { with probability } p
$$

Step 4: update:

$$
x_{i}=x_{i}+v_{i}
$$

Step 1 reflects the rush of drivers to accelerate until full speed $v_{\text {max }}$; step 2 is to prevent accidents, the driver must stop if the speed exceeds the distance that separates it from the car ahead; in step 3 are considered the different parameters on the behavior of drivers to accelerate or decelerate from which are created traffic jams; to step 4 gives the system configuration at time $t+1$ from time $t$.

\section{RNSL Model}

The NaSch model is not able to modeling the full complexity of the flow of traffic because the traffic flow is composed of various types of cars with different desired speeds, besides moving over a lane. The RNSL model generalizes the model NaSch to 2 lanes, for this, CA considered a 1-dimensional consisting of two adjacent arrays 1-dimensional size $L$ over which the NaSch model has been implemented in each arrangement and rules added to simulate the lane change.

The update of the system is carried out in two sub-steps:

1. Analyze the lane change under the new set of rules. If a vehicle meets the conditions of lane change, it is moved to the next lane transversely without moving forward. This sub-step is not possible in reality, but with the following sub-step simulation get the desired lane change.

2. Update the system according to the rules NaSch model independently in each arrangement. This sub-step setup makes use of the sub-step 1.

The lane change model is as follows:

If $k$ is the position of the $k$-th site on lane $j$. Then $k_{-j} \leq k$ denote the site on the lane $j$ that containing the nearest car to site from backward $k$; and $k_{+j}>k_{-j}$ the site on lane $j$ that containing the nearest car to site $k$ from forward. If $x_{i_{j}}$ represents the $i$-th position of the vehicle in lane $j$, then the following variables are defined: 
1. $\operatorname{gap}(i)_{j}=x_{i+1_{j}}-x_{i_{j}}-1$, the total number of empty places in front of a vehicle.

2. $\operatorname{gap}_{o}(i)_{j k}=\left(x_{i_{j}}\right)_{+k}-x_{i_{j}}-1$, the number of empty places in front of the rail $k$, to where you want to make the change.

3. $\operatorname{gap}_{o, \text { back }}(i)_{j k}=x_{i_{j}}-\left(x_{i_{j}}\right)_{-k}$, the number of sites where the closest vehicle to the rear in the lane $k$.

The RNSL model is defined by the following set of rules that are applied in parallel to each of the vehicles of the system at each time step $t$.

1. $\operatorname{gap}(i)_{j}<l$,

2. $\operatorname{gap}_{o}(i)_{j k}<l_{o}$

3. $\operatorname{gap}_{o, b a c k}(i)_{j k}<l_{\text {o.back }}$,

4. $\operatorname{rand}()<p_{\text {change }}$,

5. The vehicle changes the lane $j$ to lane $k$ placing in position $i$ of the lane $k$.

6. The system is updated by applying the rules NaSch model independently to each lane.

$l, l_{o}$ and $l_{o, b a c k}$ are parameters to decide how far they must find the next vehicle in front on the same lane, on the adjacent rail and backward adjacent rail respectively to perform the lane change. $p_{\text {change }}$ is the probability a vehicle lane change.

Figure 1 shows a heterogeneous system evolution of the RNSL model that presented platoons. Vehicles with fast speed $v_{\text {fast }}=5$ are represented by circles and slow vehicles with maximum speed $v_{\text {slow }}=2$ are represented by squares. The values of the parameters considered are $l=\min \left(v_{i}+1, v_{\text {imax }}\right)$, $l_{o}=l$ and $l_{o, \text { back }}=v_{\text {imax }}$. At the time instant $t$, a fast vehicle is in position 4 of the rail $(1)$ with a speed of $3(\approx 81 \mathrm{~km} / \mathrm{h})$. With the conditions shown in figure, the vehicle may change lanes increase its speed to $4(\approx 108 \mathrm{~km} / \mathrm{h})$ and placed in position 8 of the rail (2), however, the RNSL model does not allow lane change as $\operatorname{gap}_{o, b a c k}(4)_{12}=2=l_{\text {o,back }}$, that is, does not comply with Part 3) of the model; this has the effect the obstruction of the free flow of fast vehicles. The proposed model seeks correct this deficiency that presenting the RNSL model to allow the lane change, to thereby have a more robust and more attached to reality. 


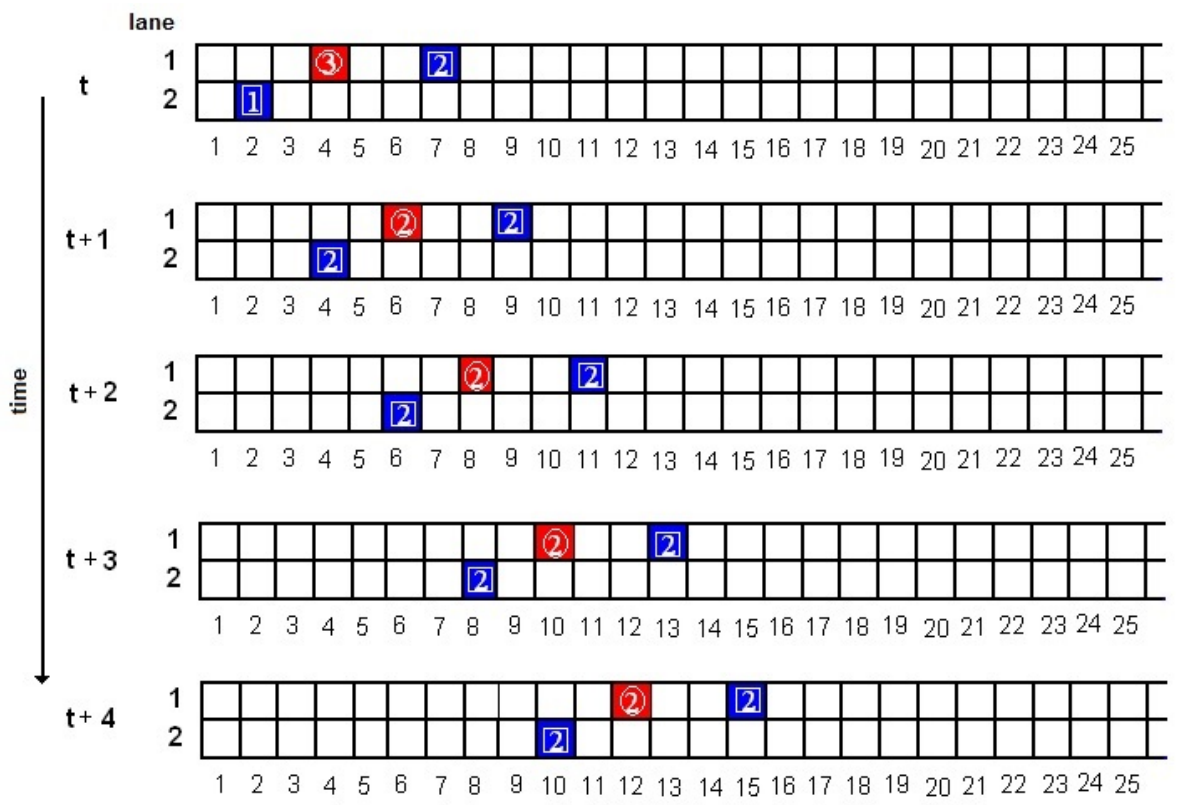

Figure 1: Displays a RNSL model evolution. Sites with brands $\bigcirc$ represent fast vehicles with maximum speed $v_{\text {fast }}=5$ and sites with brands $\square$ represent slow vehicles with maximum speed $v_{\text {slow }}=2$.

\section{$4 \quad$ DM Model}

The traffic flow model proposed by Moussa and Daoudia is a model that generalizes the Nasch model to 3 lanes similar to the model as shown RNSL. Moussa and Daoudia considered a CA 1-dimensional composed of three arrays 1-dimensional adjacent of size $L$ on which the NaSch model is implemented in each arrangement and rules that are added to simulate the lane change. The update of the system is carried out in two sub-steps:

1. Analyze the lane change under the new set of rules. If a car meets the lane change conditions, it is moved transversely to the next lane without moving forward. This sub-step is not possible in reality; nevertheless $t$ with the following sub-step is obtained from the lane change simulation desired.

2. Update the system according to the rules NaSch model independently in each arrangement. This sub-step setup makes use of the sub-step 1.

The corresponding lane change rules are defined by the following two criteria: 1) a driver needs an incentive to make a lane change and 2) a change of lane is carried out with a safety criterion. 
The lane change model is as follows:

The rules that define the lane change speed of a car $v_{i}$ are:

1. Criterion Incentive: $v_{\text {hope }_{j}}(i)>\operatorname{gap}(i)_{j}$ with $v_{\text {jope }_{j}}(i)=\min \left(v_{i}+1, v_{\text {max }}\right)$. This criterion is applied to any rail $j$ that containing the considered car.

2. For safety criterion are two cases:

(a) If the $i$-th vehicle is in the lane $j=1$ or $j=3$ and are met:

i. $\operatorname{gap}_{o}(i)_{j 2}>\operatorname{gap}(i)_{j}$,

ii. $\operatorname{gap}_{o, b a c k}(i)_{j 2}>v_{\text {max }}$,

iii. $\operatorname{rand}()<p_{\text {change }}$, with $p_{\text {change }}$ the probability parameter lane change, then the rail car changes from $j$ to rail (2) placing in position $i$ of the rail (2).

(b) If the $i$-th vehicle is in lane (2) and are met:

i. $\operatorname{gap}_{o}(i)_{2 j}>\operatorname{gap}(i)_{2}$,

ii. $\operatorname{gap}_{o, b a c k}(i)_{2 j}>v_{\max }$,

iii. $\operatorname{rand}()<p_{\text {change }}$, then the car switch to rail $(2)$ at lane $j$, placing in position $i$ of the rail $j$.

3. The system is updated by applying the rules NaSch model independently to each lane.

The following example shows a deficiency that presents the D-M model. Consider the three-lane model as shown in Figure 2. Assume that the speed of the vehicle $a$ located in the position 9 of the rail (1) is equal to $v_{\max }=5$ $(\approx 135 \mathrm{~km} / \mathrm{h})$ and the speeds of the cars $b$ and $c$ located at position 14 of the rail (1) and at position 9 of the rail $(2)$ respectively are $2(\approx 54 \mathrm{~km} / \mathrm{h})$. Since as $5=v_{\text {hope }}(8)>\operatorname{gap}(9)_{1}=4$, incentive criterion is met for the lane change for the car $a$. Must $11=\operatorname{gap}_{o}(9)_{12}>\operatorname{gap}(9)_{1}$, then meets the first part of the safety criterion, however, as $2=\operatorname{gap}_{o, b a c k}(9)_{12}<v_{\max }$, not met then the second part of the safety criterion and therefore the car $a$ remain on the rail (1) in the next time step; however, reality, a car speed equal to $135 \mathrm{~km} / \mathrm{h}$ and in these conditions can change the channel (2) without any problems. The proposed model is looking to correct this deficiency presents the D-M to allow the lane change, to thereby have a more robust 2 models nearest to reality.

\section{Proposed model for two lanes}

The proposed model which we refer to as $2 \mathrm{C}$ model consists of two sets of rules: 1) lane-changing rules and 2) displacement rules. 1) Rules lane change: 


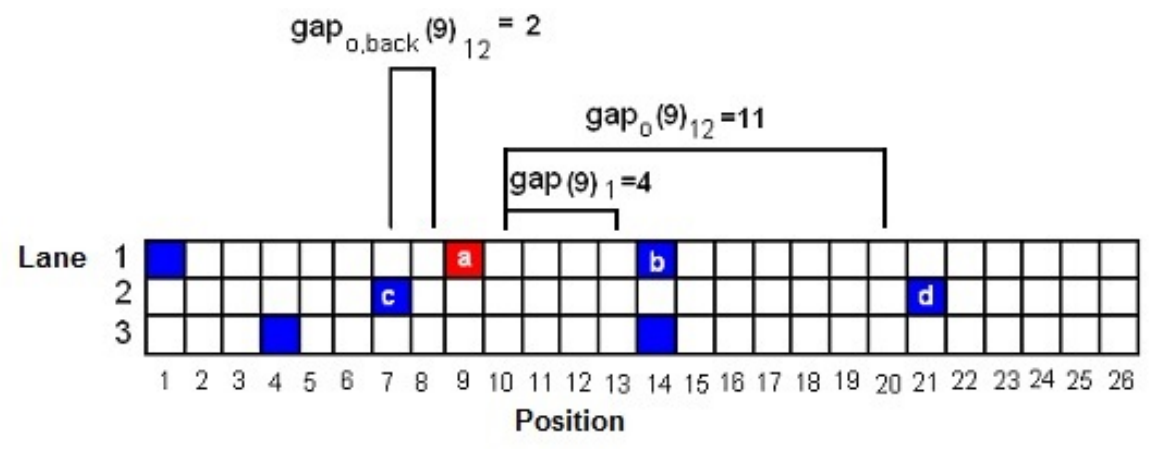

Figure 2: Displays the basic parameters for the rules of a lane change on a fast track 3 lanes in the D-M model. Colored sites are occupied by car. The letter a represents the location of the vehicle of our interest.

a) a driver needs an incentive to make a lane change and b) a lane change is performed with a safety criterion. The rules are applied in parallel to each of the vehicles of the system in each pass time step $t$, and are defined as follows:

1. Lane Change Rules:

(a) Criterion Incentive: $v_{\text {hope }_{j}}(i)>\operatorname{gap}(i)_{j}$ with $v_{\text {hope }_{j}}(i)=\min \left(v_{i}+\right.$ $\left.1, v_{\max }\right)$. This criterion is applied to lane $j$ containing the vehicle concerned.

(b) Safety criteria:

i. $\operatorname{gap}_{o}(i)_{j k}>v_{i}$,

ii. $\operatorname{gap}_{o, \text { back }}\left(i+\operatorname{gap}(i)_{j k}\right)>v_{o, b a c k}(i)_{-k}$, with $v_{o, b a c k}(i)_{-k}$ vehicle speed at the site $i_{-k}$,

(c) $\operatorname{rand}()<p_{\text {change }}$,

(d) The vehicle changes lane $j$ to $k$, placing in position $x_{i_{j}}+v_{\text {hope }_{j}}(i)$ on the lane $k$.

2. Rules of displacement: The system is updated by applying the rules NaSch model independently to each lane unmodified vehicles that made the lane change.

Figure 3 shows the evolution of a system heterogeneous of the model $2 \mathrm{C}$. At the instant of time $t$, the system configuration is equal to the time instant $t$ of Figure 1. While the RNSL model (Figure 1) are created platoons, because slow vehicles stop the free movement of fast vehicles, with the model $2 \mathrm{C}$ fast vehicles exceed to slow vehicles such platoons decreasing and achieving a greater flow vehicles. 


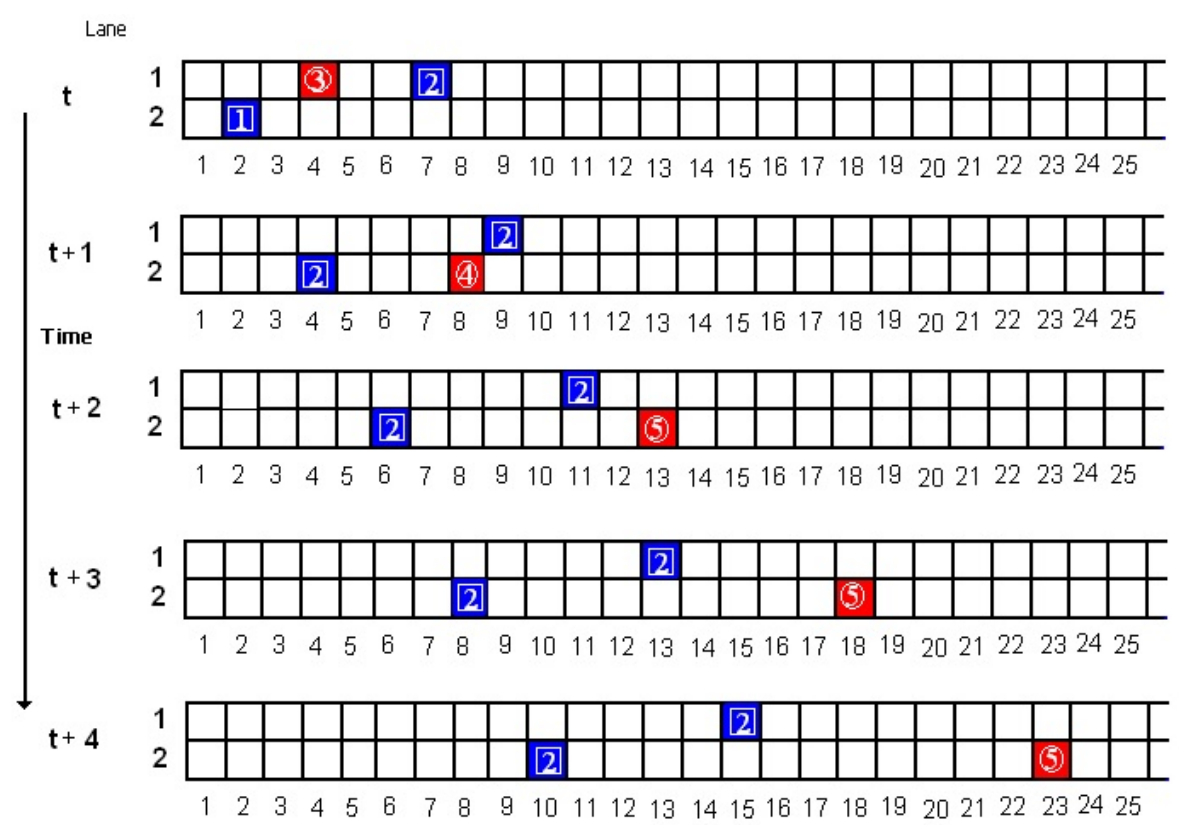

Figure 3: Displays a 2C model evolution. Sites with brands $\bigcirc$ represent fast vehicles with maximum speed $v_{\text {fast }}=5$ and sites with brands $\square$ represent the slow vehicles with maximum speed $v_{\text {slow }}=2$.

\section{Proposed model to three lanes}

The proposed model which we refer to as $3 \mathrm{C}$ consists of two sets of rules: 1 ) lane-changing rules and 2) displacement rules. Below is mention each of the rules.

1. Rules lane change:

(a) Incentive criteria: $v_{\text {hope }_{j}}(i)>\operatorname{gap}(i)_{j}$ with $v_{\text {hope }_{j}}(i)=\min \left(v_{i}+\right.$ $\left.1, v_{\max }\right)$. This criterion is applied to any rail $j$ that containing considered car.

(b) For safety criterion are two cases:

i. If the $i$-th vehicle is in the lane $j=1$ or $j=3$ and are met:

A. $\operatorname{gap}_{o}(i)_{j 2}>v_{i}$,

B. $\operatorname{gap}_{o, b a c k}\left(i+\operatorname{back}(i)_{j}\right)_{j 2}>v_{o, b a c k}(i)_{-2}$ with $v_{o, \text { back }}(i)_{-2}$ the speed of the car at the site $i_{-2}$,

C. $\operatorname{rand}()<p_{\text {change }}$, then the car changes lane $j$ to lane $(2)$, placing in position $x_{i_{j}}+v_{\text {hope }_{j}}(i)$ on the rail (2).

ii. If the $i$-th car is located in the rail (2) and are met:

A. $\operatorname{gap}_{o}(i)_{2 j}>v_{i}$, 
B. $\operatorname{gap}_{o, b a c k}\left(i+\operatorname{back}(i)_{j}\right)_{2 j}>v_{o, b a c k}(i)_{-j}$ with $v_{o, b a c k}(i)_{-j}$ the speed of the car at the site $i_{-j}$,

C. $\operatorname{rand}()<p_{\text {change }}$, then the car changes lane $j$ to lane $(2)$, placing in position $x_{i_{2}}+v_{\text {hope }_{2}}(i)$ on the lane $j$.

(c) Rules of displacement:

The system is updated by applying the rules NaSch model independently to each lane without modifying those cars that made the lane change.

\section{Simulation of the proposed models}

It was considered a fast track two and three lanes to carry out the respective comparison of the models with two different situations: a) the symmetric case, where both the slow and the fast cars can make use of all lanes, i.e., the set of rules, that define the lane change are applied equally to both types of cars; b) the asymmetric case, where fast cars can travel on any lane, while the slow cars are restricted to driving on the central lane and right side in the case of three lanes and right lane only in the case of two lanes. The simulations were performed on a lattice of size 300 sites $\approx 2.25 \mathrm{~km}$ with periodic boundary conditions. The system is heterogeneous. In the initial state, cars are randomly distributed in the lattice with zero initial velocity, with the proportion of $10 \%$ slow cars with maximum speed $v_{\text {slow }}=2$ and $90 \%$ of maximum speed fast cars $v_{\text {fast }}=4$. The lane change probability is 0.85 , and the probability for each track brake is 0.15 . The simulation results were recorded after the first 1000 time steps. We denote $\rho$ to the total system density, and $\phi$ the number of vehicles passing through a position of the lattice per unit time $T$. That is:

$$
\phi=\frac{T}{2} \sum_{t=t_{o}+1}^{t_{o}+t} n_{i, i+1}(t)
$$

where $n_{i, i+1}(t)=1$ if the movement of a car is detected among the sites $i$ and $i+1$ at iteration $t \rightarrow t+1$. A $\phi$ is known as system flow.

\section{Results}

Figure 4 shows the comparison of the fundamental diagram of the RNSL model with the $2 \mathrm{C}$ model, and the $\mathrm{D}-\mathrm{M}$ model with the $3 \mathrm{C}$ model. In $4 \mathrm{a}$ ) and $4 \mathrm{~b}$ ) shows the fundamental diagrams RNSL model with the $2 \mathrm{C}$ model applied to the symmetric and asymmetric case respectively. At 4 a) shows that $\phi_{2 C}>$ $\phi_{R N S L}$ for $\rho>0.02$. And in $\left.4 \mathrm{~b}\right) \phi_{2 C}>\phi_{R N S L}$ for $\rho>0.05$. In $4 \mathrm{c}$ ) and $4 \mathrm{~d}$ ) 

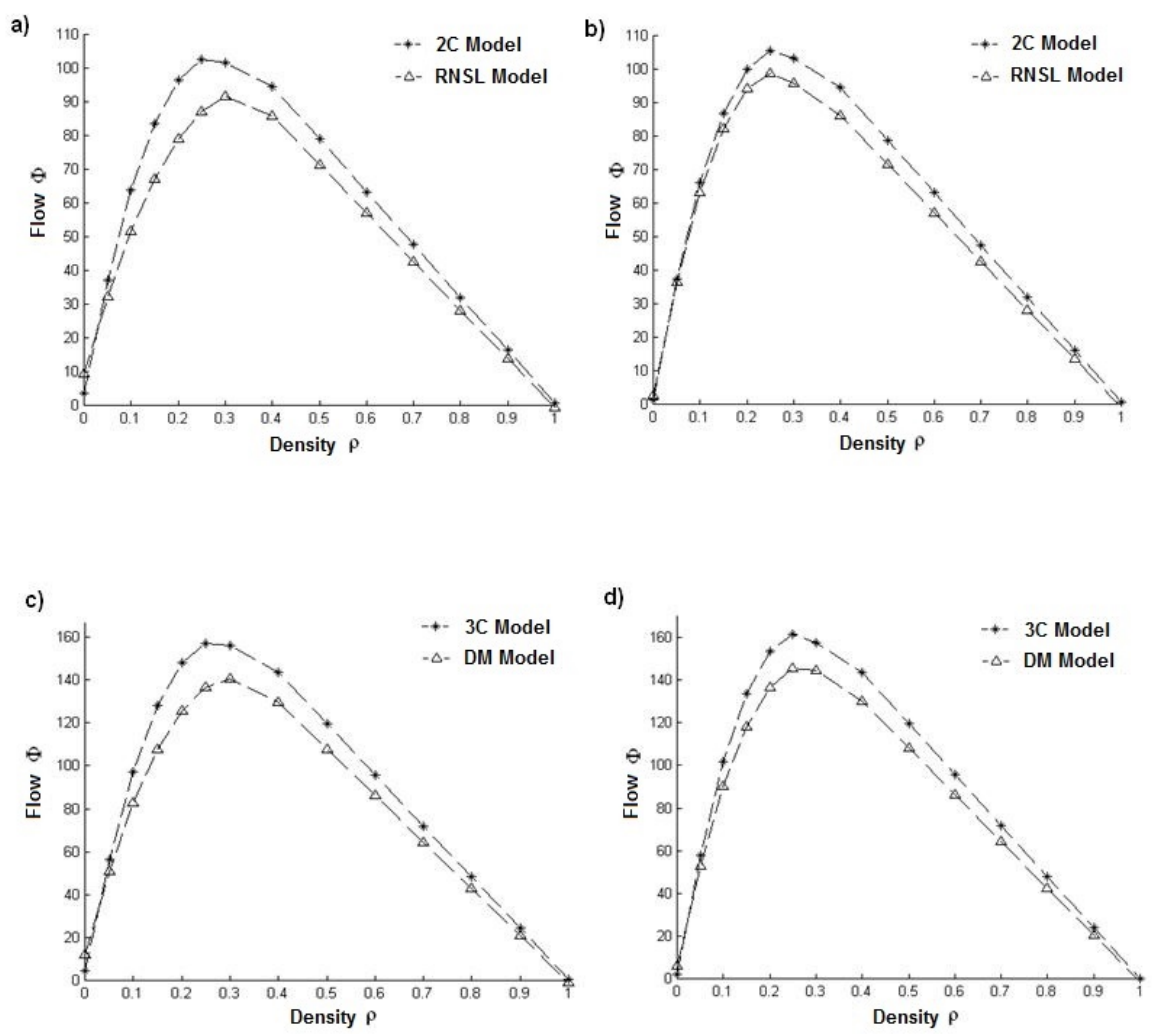

Figure 4: Comparing the fundamental diagrams between the proposed models and models RNSL and D-M respectively of two and three lanes with $p=0.15$ and $p_{\text {change }}=0.85$. In a) and b) compares the fundamental diagram of a symmetrical and asymmetrical system respectively for the case of the $2 \mathrm{C}$ model with the RNSL model. In c) and d) compares the fundamental diagram of a symmetrical and asymmetrical system respectively for the case of the $3 \mathrm{C}$ model with the D-M model.

shows the fundamental diagrams of the D-M model with the $3 \mathrm{C}$ model applied to the symmetric and asymmetric case respectively. In $4 \mathrm{c}$ ) and $4 \mathrm{~d}$ ) shows the fundamental diagrams of the D-M model with the 3C model applied to almost symmetrical and asymmetrical respectively. In $4 \mathrm{c}$ ) shows that $\phi_{3 C}>\phi_{D M}$ for $\rho=0.02$. And in $4 \mathrm{~d}) \phi_{3 C}>\phi_{D M}$ for $\rho>0.01$.

\section{Conclusions}

There have been two models of traffic flow multi-lane, one associated with the flow of traffic to two lanes and one of three lanes, to which we refer to as model $2 \mathrm{C}$ and $3 \mathrm{C}$ respectively. The $2 \mathrm{C}$ model was compared with the model RNSL, 
and 3C model was compared with the model D-M, via both fundamental diagram. It is observed that the traffic flow of the proposed models is increased compared with the model D-M and RNSL, this is because the proposed models allow lane changes that may occur in the real flow and that with the RNSL and D-M models is not possible causing a greater amount of platoons and therefore decreasing traffic flow.

ACKNOWLEDGEMENTS. The authors would like to thank the Instituto Politécnico Nacional (Secretaría Académica, COFAA, SIP, ESCOM and CIDETEC) for their economical support to develop this work.

\section{References}

[1] K. Daoudia, N. Moussa, Numerical simulations of a three-lane traffic model using cellular automata, Chinese journal of physic, Vol. 41, No. 6, December 2008, pp. 123-127.

[2] K. Nagel, M. Schreckenberg, A cellular automaton model for freeway traffic, J. Phys. I. France 2, December 1992, pp. 2221-2229.

[3] M. Rickert, K. Nagel, M. Schreckenberg, A. Latour. Two lane traffic simulations using cellular automata. Physica A: Statistical and theoretical physics, vol. 231, issue 4, 1, October 1996, pp. 534-550.

[4] R. Mu, T. Yamamoto, An analysis on mixed traffic flow of conventional passenger cars and microcars using a cellular automata model. Procedia - social and behavioral sciences, vol. 43, 2012, pp. 457-465.

[5] S. C. Lo, C. H. Hsu, Cellular automata simulation for mixed manual and automated control traffic . Mathematical and computer modelling, vol. 51, Issues 7-8, April 2010, pp. 1000-1007.

[6] S. Darbha, K.R. Rajagopal, V. Tyagi, A review of mathematical models for the flow of traffic and some recent results. Nonlinear analysis: Theory, methods \& applications, vol. 69, issue 3, 1 August 2008, pp. 950-970.

Received: April 21, 2013 